\title{
Review Article \\ Thyroidectomy and Lymph Node Dissection in Papillary Thyroid Carcinoma
}

\author{
Yasuhiro Ito and Akira Miyauchi \\ Departments of Surgery, Kuma Hospital, 8-2-35, Shimoyamate-dori, Chuo-ku, Kobe City 650-0011, Japan \\ Correspondence should be addressed to Yasuhiro Ito, ito01@kuma-h.or.jp \\ Received 2 August 2010; Accepted 14 October 2010 \\ Academic Editor: Ashok Shaha
}

Copyright ( $) 2011$ Y. Ito and A. Miyauchi. This is an open access article distributed under the Creative Commons Attribution License, which permits unrestricted use, distribution, and reproduction in any medium, provided the original work is properly cited.

Papillary carcinoma is a prominent malignancy originating from follicular cells. This disease generally shows an indolent character, but patients demonstrating certain clinicopathological features have a dire prognosis. At present, Western countries adopted almost routine total thyroidectomy with radioactive iodine (RAI) ablation, while limited thyroidectomy with extensive prophylactic lymph node dissection has traditionally been performed for most patients in Japan. Recently, accurate evaluation of carcinoma stage can be performed on preoperative imaging studies, especially on ultrasonography. It is therefore important to treat papillary carcinoma patients depending on clinicopathological features rather than in a stereotyped fashion. In this paper, appropriate extension of thyroidectomy and lymph node dissection is discussed based on Western and recently published Japanese guidelines and the experience in Kuma Hospital.

\section{Introduction}

Papillary carcinoma is the most common malignancy arising from thyroid follicular cells. In Western countries, papillary carcinoma comprises $85 \%$ in whites and $72 \%$ in blacks $[1,2]$. According to an investigation of the Japanese Society of Thyroid Surgeons (JSTS) in 2004, the incidence of papillary carcinoma in Japan was 93\%, which was higher than that in Western countries, possibly because of sufficient amounts of iodine in the Japanese diet. Generally, it has an indolent character, but cases showing certain clinicopathological features are progressive and show a dire prognosis. Frequent multiplicity in the thyroid and metastasis to the regional lymph nodes is prominent biological characteristics of papillary carcinoma. Therefore, not only the extent of thyroidectomy but also that of lymph node dissection remains controversial.

In Western countries, total (or near total) thyroidectomy has been almost routinely performed because the serum thyroglobulin level can be used as a marker of recurrence or persistent disease, and radioactive iodine (RAI) ablation can be performed as an adjuvant therapy. Moreover, RAI therapy can be immediately performed when the thyroglobluin level becomes elevated or recurrence is clinically detected. In Japan, however, limited thyroidectomy such as subtotal thyroidectomy and lobectomy with isthmectomy has been traditionally adopted as the standard. This is partially because the capacity to perform RAI therapy is limited due to legal restrictions, and RAI therapy is not considered cost effective by the healthcare system in Japan. Furthermore, it is also because Japanese endocrine surgeons are empirically aware that most papillary carcinomas are indolent and their prognoses are generally excellent. Limited thyroidectomy decreases the incidence of severe complications such as bilateral recurrent laryngeal paralysis and persistent hypoparathyroidism, and patients may not require the administration of L-thyroxine.

In contrast, lymph node dissection is not actively performed in Western countries. In Japan, however, extensive prophylactic lymph node dissection not only in the central but also lateral compartment has been widely adopted. This is because the organ to which papillary carcinoma most frequently recurs is the lymph node [3], and prophylactic lymph node dissection can decrease the recurrence rate of carcinoma.

From early in the 1990s, the elevated resolution power of ultrasonography has facilitated the accurate evaluation of 
TABLE 1: Indication for total thyroidectomy for patients with papillary carcinoma in various guidelines and in our department.

\begin{tabular}{|c|c|}
\hline Kuma Hospital & $\begin{array}{l}\text { All patients except T1N0M0 or microcarcinoma extending only to muscles mucosal layer of the } \\
\text { esophagus and recurrent laryngeal nerve }\end{array}$ \\
\hline JSTS/JAES & $\begin{array}{l}\text { Strongly recommended } \longrightarrow \text { Tumor size }>5 \mathrm{~cm} \text {, extrathyroid extension to the trachea or esophagus, } \\
\text { large number of clinical lymph node metastasis, lymph node metastasis }>3 \mathrm{~cm} \text {, and distant metastasis } \\
\text { Moderately recommended } \longrightarrow \text { Tumor size }>4 \mathrm{~cm} \text {, clinical lymph node metastasis }\end{array}$ \\
\hline ATA & All patients except low-risk microcarcinoma \\
\hline BTA & $\begin{array}{l}\text { Most patients, especially for those with tumor size }>1 \mathrm{~cm} \text {, multifocal disease, extrathyroid extension, } \\
\text { familial disease, clinical lymph node metastasis, and radiation history }\end{array}$ \\
\hline NCCN & $\begin{array}{l}\text { Age }<15 \text { yrs or }>45 \text { yrs, radiation history, distant metastasis, bilateral nodularity, extrathyroidal } \\
\text { extension, Tumor size }>4 \mathrm{~cm} \text {, clinical lymph node metastasis, and aggressive variant (however, also for } \\
\text { other cases, total thyroidectomy is the most common) }\end{array}$ \\
\hline
\end{tabular}

thyroid carcinoma by demonstrating the size and location of primary lesions and lymph node metastases. Based on the prognostic data of patients who underwent surgery in the era of routine ultrasonography, it may be the time to revise the standards regarding the optimal extent of thyroidectomy and lymph node dissection for papillary carcinoma. In this paper, we compare the strategies of thyroidectomy and lymph node dissection among Western and Japanese guidelines as well as our experience in order to consider the most appropriate surgical designs for papillary carcinoma patients.

\section{Thyroidectomy}

Total thyroidectomy is almost routinely recommended by Western guidelines such as those of National Comprehensive Cancer Network (NCCN), ATA, and BTA [4-6].

As indicated in the introduction, limited thyroidectomy has been more widely adopted than total thyroidectomy in Japan because of the limited capacity to perform RAI therapy, and the policy of Japanese endocrine surgeons that limited thyroidectomy is adequate for most papillary carcinoma patients. In the Japanese guideline recently published, it is indicated that, for patients with T1N0M0 according to UICC TNM classification system, hemithyroidectomy is acceptable and adequate [7]. Indeed, one Japanese study showed that 10-year disease-free survival (DFS) rates of 2638 solitary T1N0M0 patients were excellent at $97 \%$ even though $60 \%$ of these patients underwent limited thyroidectomy [8]. The incidence of recurrence to the remnant thyroid in patients who underwent limited thyroidectomy was only $1 \%$. Distant metastasis was detected only in 4 patients $(0.2 \%)$. Two patients died of carcinoma due to the growth of local or distant recurrence. However, patients who underwent total thyroidectomy are easier to follow than those receiving limited thyroidectomy because, as indicated above, thyroglobulin level can be a marker of recurrence, and RAI therapy can be immediately performed for carcinoma recurrence. Therefore, total thyroidectomy should be the first line for patients with high-risk features predicting poor diseasefree and cause-specific survival rates. In our guideline, total thyroidectomy is strongly recommended for patients having tumor size larger than $5 \mathrm{~cm}$, large number of clinical lymph node metastases detected on imaging studies, lymph node larger than $3 \mathrm{~cm}$, significant extrathyroid extension, or distant metastasis at surgery [7]. Such patients are regarded as high risk and considered likely to show recurrence to local and/or distant organs. Furthermore, our guideline also described that total thyroidectomy is preferable also for patients having tumor larger than $4 \mathrm{~cm}$ or clinical lymph node metastasis.

Currently, our indication for total thyroidectomy is wider than that in the Japanese guideline but still narrower than that in Western guidelines. We recommend total thyroidectomy for all patients except low-risk patients such as solitary T1N0M0 patients and those with microcarcinoma extending only to the muscles, muscle layer of the esophagus, or recurrent laryngeal nerve. Indeed, the incidence of total thyroidectomy is increasing in our department mainly because of the ease of postoperative followup.

Table 1 summarizes the indications for total thyroidectomy in Western and Japanese guidelines and in our hospital.

\section{Lymph Node Dissection}

Regional lymph nodes are located in the three compartments; central, lateral, and mediastinal compartments. Although one study recommended prophylactic mediastinal dissection in papillary carcinoma showing contralateral lateral node metastasis [9], generally this compartment is dissected only when radiologic evidence of metastasis is detected. In this paper, indications for dissection of central and lateral compartments are described.

3.1. Central Compartment. Of the three compartments, the central compartment is the most adjacent to the thyroid. This compartment can be dissected without extension of the wound for thyroidectomy or exacerbation of postoperative complications such as neck discomfort and pain. In an investigation of 5805 patients who underwent central node dissection, the positive predictive value and specificity of preoperative ultrasonography were high at $92 \%$ and $98 \%$, respectively, but negative predictive value and sensitivity were terribly low at $37 \%$ and $12 \%$, respectively [3]. This indicates that, of patients diagnosed as negative on ultrasonography, $63 \%$ are diagnosed as having positive lymph nodes in this compartment on pathological examination. 
TABLE 2: Indications for prophylactic central node dissection for patients with papillary carcinoma in various guidelines and in our department.

\begin{tabular}{ll}
\hline Kuma Hospital & Routinely recommended \\
JSTS/JAES & Routinely recommended \\
ATA & May be performed for T3 or T4 patients \\
BTA & Male gender, age $>45$ yrs, tumor size $>4 \mathrm{~cm}$, and extracapsular or extrathyroidal disease \\
& Can be considered for patients with age $<15$ yrs or $>45$ yrs, \\
NCCN & radiation history, distant metastasis, bilateral nodularity, extrathyroidal \\
& extension, tumor size $>4 \mathrm{~cm}$, and aggressive variant \\
\hline
\end{tabular}

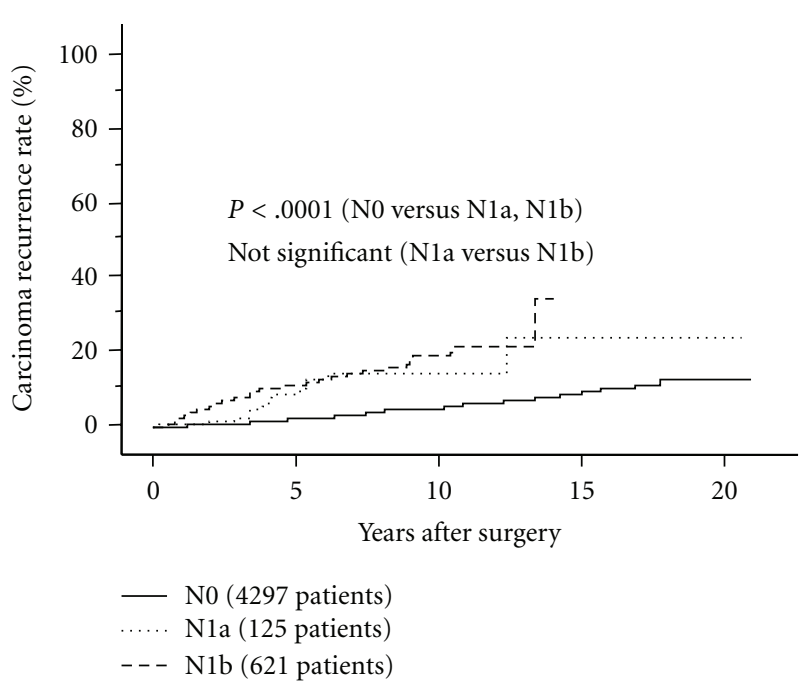

Figure 1: Carcinoma recurrence rates for patients who did not show clinical node metastasis (N0), those with clinical central node metastasis (N1a), and those with lateral node metastasis (N1b). Patients having significant extrathyroid extension were deleted from the series.

This is possibly because the air-filled trachea, clavicle, and menubrium of the sternum disrupt the accurate depiction on ultrasonography.

Patients having clinical node metastasis not only to the lateral but also to the central compartment are more likely to show carcinoma recurrence than patients without clinical node metastasis (Figure 1) [10]. Therefore, careful therapeutic node dissection should be performed for such patients. However, there are discrepant data on improving the prognosis of central node dissection [11-18], and there are no studies comparing prognoses between patients who underwent prophylactic central node dissection and did not undergo that. Therefore, it is still debatable whether prophylactic central node dissection is of clinical significance.

Western guidelines do not recommend routine prophylactic central node dissection [4-6]. In contrast, the Japanese guideline recommends routine prophylactic central node dissection [7]. This is because, as indicated above, central node metastasis is difficult to evaluate preoperatively, and reoperation for recurrence to this compartment may cause severe complications such as recurrent laryngeal nerve injury and persistent hypoparathyroidism. Our department has also
TABLE 3: Relationship between metastasis to the central and lateral compartments in 3940 patients.

\begin{tabular}{lcccc}
\hline & & \multicolumn{3}{c}{ Lateral node metastasis (\%) } \\
& & Negative & Positive & Total \\
\hline Central node & Negative & 21 & $\mathbf{1 1}$ & 32 \\
metastasis (\%) & Positive & $\mathbf{1 5}$ & 53 & 68 \\
\hline & & 36 & 64 & 100 \\
\hline
\end{tabular}

traditionally adopted the same procedure for central node dissection as outlined in the Japanese guideline. The Japanese guideline also indicates that in patients who undergo hemithyroidectomy, the pretracheal and peritracheal nodes ipsilateral to the primary lesion as well as the Delphian nodes should be dissected, while dissection of peritracheal nodes contralateral to primary lesion is not mandatory. We agree with this guideline because, according to our data, central node dissection in the contralateral lobe did not improve the prognosis of patients with microcarcinoma [8].

The recommended management of central compartment dissection in the Japanese guideline and in our department is in sharp contrast to that in Western guidelines. Table 2 summarizes the strategies in the guidelines and in our department.

3.2. Lateral Compartment. The lateral compartment is more distant from the thyroid than the central compartment. In order to dissect this compartment, wound extension is necessary and, therefore, we have considered that papillary carcinoma initially metastasizes to the central compartment and, thereafter, metastasizes to the lateral compartment. However, as shown in Table 3, the incidence of centralnegative but lateral-positive patients was similar to that of central-positive but lateral-negative patients $[3,19]$. It is thus suggested that papillary carcinoma shows similar incidences of initially metastasizing to the central and lateral compartments.

For patients showing clinical lateral node metastasis (N1b), therapeutic lateral node dissection is mandatory. Clinical lateral node metastasis is an important and independent prognostic factor $[3,20]$. Especially, patients having metastatic nodes larger than $3 \mathrm{~cm}$ or extranodal tumor extension have a significantly worse disease-free survival and cause-specific survival $[10,21]$. Thus, careful node dissection 
TABLE 4: Relationship between lateral node metastasis and tumor size in 1231 patients who were diagnosed as negative for metastasis on preoperative ultrasonography but underwent prophylactic lateral node dissection.

\begin{tabular}{lcccc}
\hline \multicolumn{5}{c}{ Number of metastases $(\%)$} \\
Tumor size $(\mathrm{cm})$ & 0 & $1-4$ & 5 or more & Total \\
\hline 1.0 or less & $78(59.5)$ & $43(32.8)$ & $10(7.6)$ & 131 \\
$1.1-2.0$ & $191(47.9)$ & $143(35.8)$ & $65(16.3)$ & 399 \\
2.1 or more & $172(24.5)$ & $299(42.7)$ & $230(32.8)$ & 701 \\
\hline
\end{tabular}

TABLE 5: Indications for prophylactic lateral node dissection for patients with papillary carcinoma in various guidelines and in our department.

\begin{tabular}{ll}
\hline Kuma Hospital & Tumor size $>3 \mathrm{~cm}$, extrathyroid extension \\
JSTS/JAES & $\begin{array}{l}\text { Not determined, although its significance } \\
\text { in reducing recurrence is recognized }\end{array}$ \\
ATA & Not recommended \\
BTA & Not recommended \\
NCCN & Not recommended \\
\hline
\end{tabular}

is necessary in order to prevent recurrence at least to the compartment that had previously been dissected.

However, the indications for prophylactic lateral node dissection remain debatable. Diagnostic accuracy for lateral node metastasis on ultrasonography is somewhat better than that for central node metastasis, but the negative predictive value and sensitivity remain low at $43 \%$ and $29 \%$, respectively [3]. Furthermore, the incidence of lateral node metastasis for cases that are not preoperatively diagnosed as negative increases with tumor size, and about $75 \%$ of patients with primary lesions larger than $2 \mathrm{~cm}$ demonstrated latent metastasis in this compartment (Table 4) [22]. If prophylactic lateral node dissection is not performed, metastatic nodes in this compartment remain undissected at a very high incidence. Since Japanese endocrine surgeons are empirically aware of the high incidence of node metastasis to the lateral compartment, they actively perform prophylactic lateral node dissection, although none of the Western guidelines recommend this [4-6]. However, it is a separate issue whether these latent metastatic nodes become a clinical issue that later affects patient prognosis. Indeed, previous studies, including those from Japan, showed discrepant results for the significance of lateral node dissection [22-30]. Based on these, Japanese guideline recognizes that prophylactic lateral node dissection decreases the risk of recurrence and improves disease-free survival but does not clearly state its indication. Our department recommends prophylactic lateral node dissection for patients having a primary lesion larger than $3 \mathrm{~cm}$ or significant extrathyroid extension, because these patients show a significantly worse lymph node metastasis-free survival even though they underwent prophylactic lateral node dissection (Figures 2(a) and 2(b)) [22]. Another department in Japan showed that prophylactic lateral node dissection is preferable for patient having tumors greater than $4 \mathrm{~cm}$ or with distant metastasis [28]. Indications

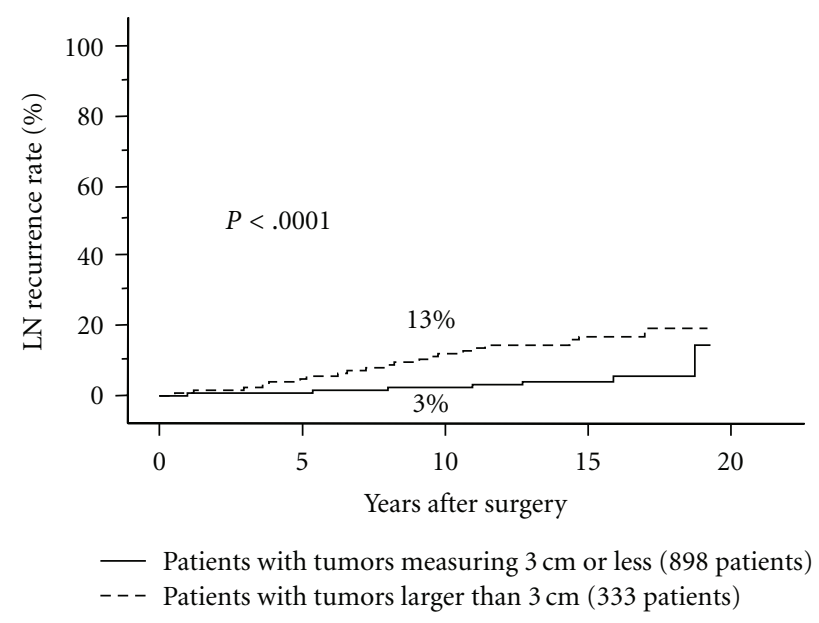

(a)

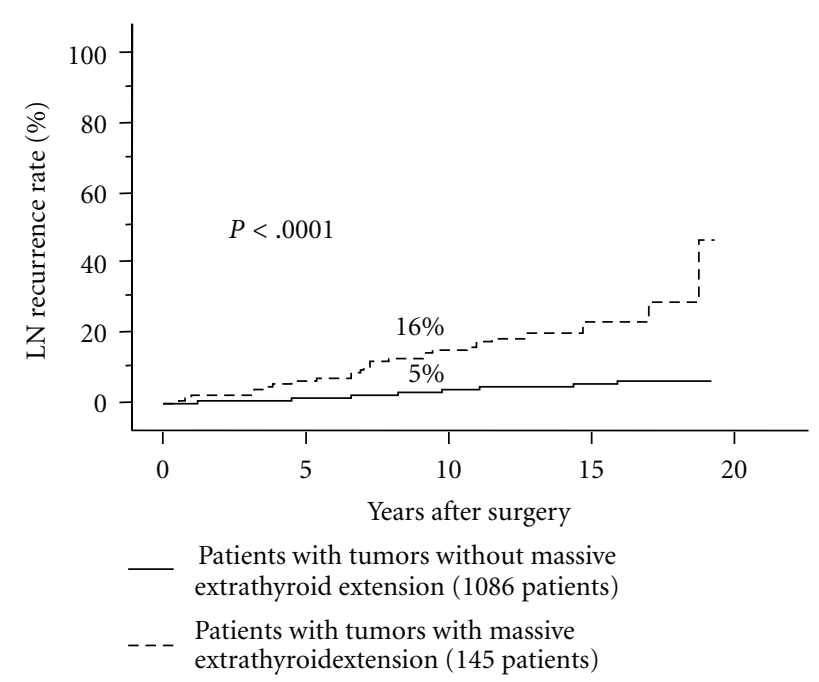

(b)

FIGURE 2: (a) Lymph node (LN) recurrence rates for patients with tumors $3 \mathrm{~cm}$ or larger and those with tumors less than $3 \mathrm{~cm}$ who underwent central and prophylactic lateral node dissection. (b) Lymph node (LN) recurrence rates for patients with and without significant extrathyroid extension who underwent central and prophylactic lateral node dissection.

of prophylactic lateral node dissection based on Western guidelines, Japanese guideline, and our department are summarized in Table 5.

\section{Conclusions}

Recently, in Japan, there has been a developing opinion that total thyroidectomy would be preferable for papillary carcinoma patients demonstrating high-risk features, and prophylactic lateral node dissection is unnecessary for lowrisk patients. In contrast, routine RAI ablation after total thyroidectomy for low-risk patients has come under review in Western countries, and in the BTA guideline, risk of second malignancy is described, indicating that routine total 
thyroidectomy for low-risk patients may become less significant [6]. We have an impression that strategies for treatment of thyroid carcinoma, including papillary carcinoma, in Western countries and Japan are coming closer to each other.

Due to the high resolution power of ultrsonography, evaluation of primary lesions, including size, number and location, and lymph node metastases, can be accurately performed. Under such circumstances, we consider that the treatment of papillary carcinoma should be determined on a case-by-case basis rather than in a stereotyped fashion. Needless to say, total thyroidectomy is mandatory for highrisk patients predicted to develop carcinoma recurrence in high incidences. It is, however, doubtful whether routine total thyroidectomy with RAI ablation is appropriate for the treatment of papillary carcinoma. For example, it is now evident that total thyroidectomy is not needed for T1N0M0 carcinoma unless there are no pathological lesions in the contralateral lobe on ultrasonography [8]. The indications for total thyroidectomy in the Japanese guideline are narrower than those in Western guidelines. In our department, the prevalence of total thyroidectomy is increasing, but the indications are still stricter than those in Western guidelines. This is based on our policy that low-risk patients show an excellent prognosis even though they undergo limited thyroidectomy.

Regarding lymph node metastasis, patients having clinical node metastasis detectable on ultrasonography are likely to show recurrence even when they undergo therapeutic lymph node dissection. Unfortunately, the incidence of recurrence to the compartments that had previously been dissected is similar to that of recurrence to the compartment that had not been dissected [3]. Therefore, very careful node dissection is necessary for such patients.

It is important to note that ultrasonography very often overlooks lymph node metastasis, and the indications for prophylactic node dissection come into question. The Japanese guidelines and our department policy recommend routine central node dissection, which is in sharp contrast to Western guidelines. In our opinion, this should be routinely performed in order to prevent recurrence to this compartment. Reoperation for recurrence to this compartment may induce severe complications such as persistent hypoparathyroidsm and recurrent laryngeal injury. The Japanese guideline does not clearly describe the indications for prophylactic lateral node dissection although the guideline recognizes the effectiveness of this approach to improving disease-free survival. This may reflect the fact that almost routine lateral node dissection was performed in the past. However, our department recommends prophylactic lateral node dissection for patients having tumors greater than $3 \mathrm{~cm}$ or significant extrathyroid extension. Although there are no comparative data, such patients show a high incidence of lymph node recurrence even though they undergo prophylactic lateral node dissection. Western guidelines do not recommend prophylactic lateral node dissection, which may be because of a difference in the lymph node recurrence rates considered acceptable by Western countries and in our department.
In summary, we described the extent of thyroidectomy and lymph node dissection recommended by the Japanese guideline and our department and compared these with those outlined in Western guidelines. We expect that treatment strategies in Western countries and Japan will become closer to each other in the future, providing the optimal treatment for patients around the world.

\section{References}

[1] P. Correa and V. W. Chen, "Endocrine gland cancer," Cancer, vol. 75, no. 1, pp. 338-352, 1995.

[2] D. M. Parkin, S. L. Whelan, and J. Ferlay, Cancer Incidence in Five Continents, vol. 8, IARC Scientific, Lyon, France, 2002.

[3] Y. Ito and A. Miyauchi, "Prognostic factors and therapeutic strategies for differentiated carcinomas of the thyroid," Endocrine Journal, vol. 56, no. 2, pp. 177-192, 2009.

[4] National Comprehensive Cancer Network, "NCCN clinical practice guidelines in oncology. Thyroid carcinoma. V. 1," 2007, http://www.nccn.org/professionals/physician_gls/PDF/ thyroid/pdf.

[5] D. S. Cooper, G. M. Doherty, B. R. Haugen et al., "Revised American thyroid association management guidelines for patients with thyroid nodules and differentiated thyroid cancer," Thyroid, vol. 19, no. 11, pp. 1167-1214, 2009.

[6] British Thyroid Association, "Guidelines for the management of thyroid cancer. Second ed," 2007, http://www.british-thyroid-association.org/news/Docs/Thyroid_cancer_guidelines 2007.pdf.

[7] Guidelines for Management of Thyroid Tumor-The Japanese Society of Thyroid Surgery - The Japanese Society of Endocrine Surgeons, Kanehara Press, Tokyo, Japan, 2010.

[8] Y. Ito, H. Masuoka, M. Fukushima et al., "Excellent prognosis of patients with solitary T1N0M0 papillary thyroid carcinoma who underwent thyroidectomy and elective lymph node dissection without radioiodine therapy," World Journal of Surgery, vol. 34, no. 6, pp. 1285-1290, 2010.

[9] A. Sugenoya, K. Asanuma, K. Shingu et al., "Clinical evaluation of upper mediastinal dissection for differentiated thyroid carcinoma," Surgery, vol. 113, no. 5, pp. 541-544, 1993.

[10] Y. Ito, M. Fukushima, C. Tomoda et al., "Prognosis of patients with papillary thyroid carcinoma having clinically apparent metastasis to the lateral compartment," Endocrine Journal, vol. 56, no. 6, pp. 759-766, 2009.

[11] L.-E. Tisell, B. Nilsson, J. Mölne et al., "Improved survival of patients with papillary thyroid cancer after surgical microdissection," World Journal of Surgery, vol. 20, no. 7, pp. 854-859, 1996.

[12] G. F. W. Scheumann, O. Gimm, G. Wegener, H. Hundeshagen, H. Dralle, and B. Cady, "Prognostic significance and surgical management of locoregional lymph node metastases in papillary thyroid cancer," World Journal of Surgery, vol. 18, no. 4, pp. 559-568, 1994.

[13] J. A. Pereira, J. Jimeno, J. Miquel et al., "Nodal yield, modality, and recurrence after central neck dissection for papillary thyroid cancer," Surgery, vol. 138, no. 6, pp. 1095-1100, 2005.

[14] N. Wada, Q.-Y. Duh, K. Sugino et al., "Lymph node metastasis from 259 papillary thyroid microcarcinomas: frequency, pattern of occurrence and recurrence, and optimal strategy for neck dissection," Annals of Surgery, vol. 237, no. 3, pp. 399407, 2003.

[15] D. Simon, P. E. Goretzki, J. Witte, and H. D. Röher, "Incidence of regional recurrence guiding radicality in differentiated 
thyroid carcinoma," World Journal of Surgery, vol. 20, no. 7, pp. 860-866, 1996.

[16] N. Sato, M. Oyamatsu, Y. Koyama, I. Emura, Y. Tamiya, and K. Hatakeyama, "Do the level of nodal disease according to the TNM classification and the number of involved cervical nodes reflect prognosis in patients with differentiated carcinoma of the thyroid gland?" Journal of Surgical Oncology, vol. 69, no. 3, pp. 151-155, 1998.

[17] M. D. Shah, F. T. Hall, S. J. Eski, I. J. Witterick, P. G. Walfish, and J. L. Freeman, "Clinical course of thyroid carcinoma after neck dissection," Laryngoscope, vol. 113, no. 12, pp. 2102 2107, 2003.

[18] Y. Ito, T. Jikuzono, T. Higashiyama et al., "Clinical significance of lymph node metastasis of thyroid papillary carcinoma located in one lobe," World Journal of Surgery, vol. 30, no. 10, pp. 1821-1828, 2006.

[19] Y. Ito and A. Miyauchi, "Lateral lymph node dissection guided by preoperative and intraoperative findings in differentiated thyroid carcinoma," World Journal of Surgery, vol. 32, no. 5, pp. 729-739, 2008.

[20] Y. Ito, C. Tomoda, T. Uruno et al., "Ultrasound-detectable and anatomopathologically-detectable node metastasis in the lateral compartment as indicators of worse relapse-free survival in patients with papillary thyroid carcinoma," World Journal of Surgery, vol. 29, no. 7, pp. 917-920, 2005.

[21] I. Sugitani, N. Kasai, Y. Fujimoto, and A. Yanagisawa, "A novel classification system for patients with PTC: addition of the new variables of large ( $3 \mathrm{~cm}$ or greater) nodal metastases and reclassification during the follow-up period," Surgery, vol. 135, no. 2, pp. 139-148, 2004.

[22] Y. Ito, T. Higashiyama, Y. Takamura et al., "Risk factors for recurrence to the lymph node in papillary thyroid carcinoma patients without preoperatively detectable lateral node metastasis: validity of prophylactic modified radical neck dissection," World Journal of Surgery, vol. 31, no. 11, pp. 20852091, 2007.

[23] H. C. Davidson, B. J. Park, and J. T. Johnson, "Papillary thyroid cancer: controversies in the management of neck metastasis," Laryngoscope, vol. 118, no. 12, pp. 2161-2165, 2008.

[24] G. Balázs, F. Gyory, G. Lukács, and S. Szakáll, "Long-term follow-up of node-positive papillary thyroid carcinomas," Langenbeck's Archives of Surgery, vol. 383, no. 2, pp. 180-182, 1998.

[25] N. R. Caron, Y. Y. Tan, J. B. Ogilvie et al., "Selective modified radical neck dissection for papillary thyroid cancer - Is level I, II and V dissection always necessary?" World Journal of Surgery, vol. 30, no. 5, pp. 833-840, 2006.

[26] N. Bhattacharyya, "Surgical treatment of cervical nodal metastases in patients with papillary thyroid carcinoma," Archives of Otolaryngology, vol. 129, no. 10, pp. 1101-1104, 2003.

[27] S. Turanli, "Is the type of dissection in lateral neck metastasis for differentiated thyroid carcinoma important?" Otolaryngology, vol. 136, no. 6, pp. 957-960, 2007.

[28] I. Sugitani, Y. Fujimoto, K. Yamada, and N. Yamamoto, "Prospective outcomes of selective lymph node dissection for papillary thyroid carcinoma based on preoperative ultrasonography," World Journal of Surgery, vol. 32, no. 11, pp. 2494-2502, 2008.

[29] S. Noguchi, N. Murakami, H. Yamashita, M. Toda, and H. Kawamoto, "Papillary thyroid carcinoma: modified radical neck dissection improves prognosis," Archives of Surgery, vol. 133, no. 3, pp. 276-280, 1998.

[30] N. Sato, M. Oyamatsu, Y. Koyama, I. Emura, Y. Tamiya, and K. Hatakeyama, "Do the level of nodal disease according to the
TNM classification and the number of involved cervical nodes reflect prognosis in patients with differentiated carcinoma of the thyroid gland?" Journal of Surgical Oncology, vol. 69, no. 3, pp. 151-155, 1998. 


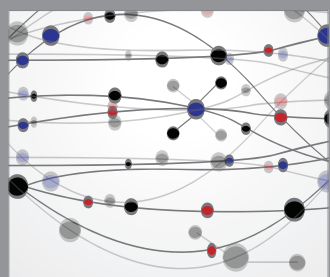

The Scientific World Journal
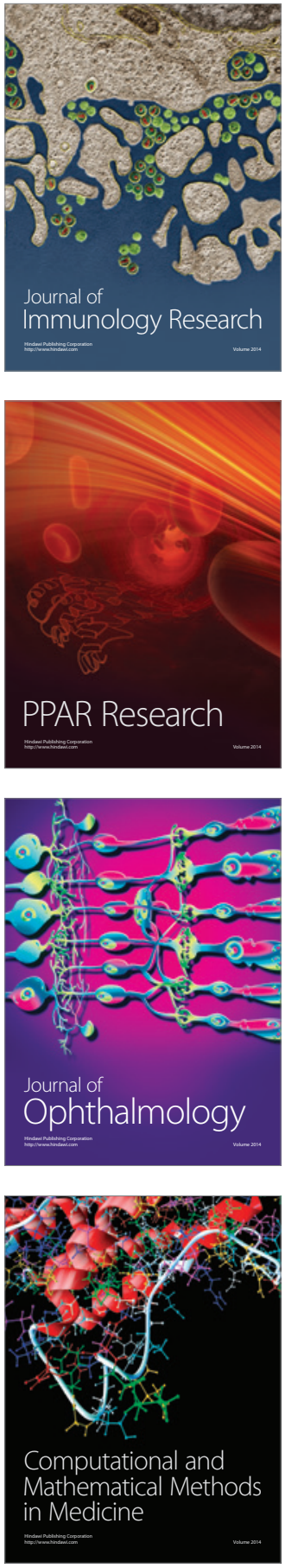

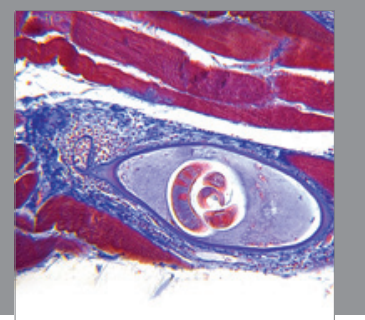

Gastroenterology

Research and Practice
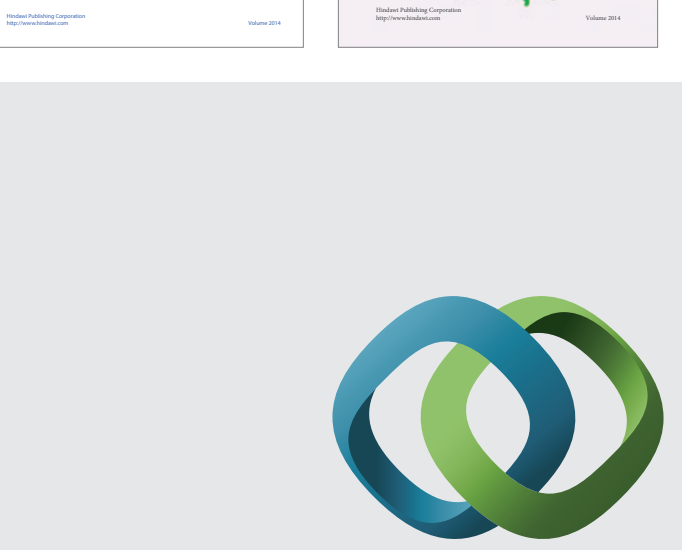

\section{Hindawi}

Submit your manuscripts at

http://www.hindawi.com
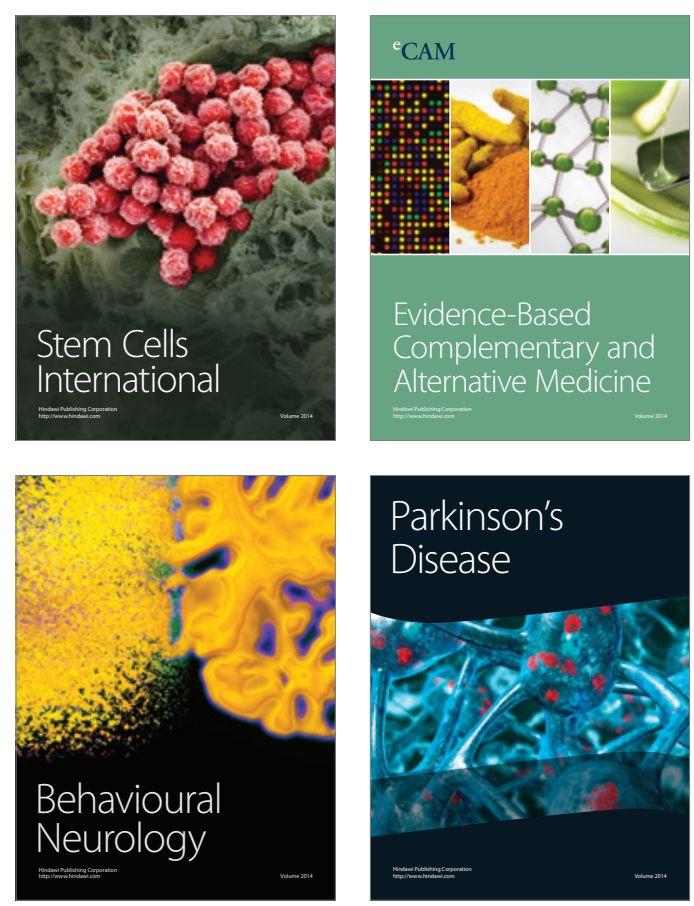

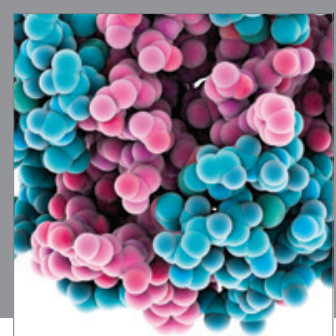

Journal of
Diabetes Research

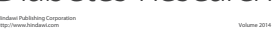

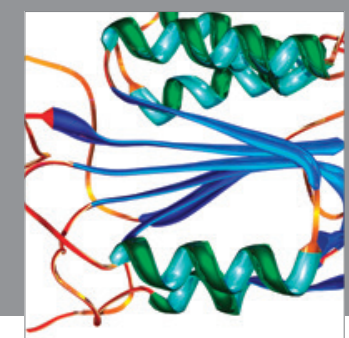

Disease Markers
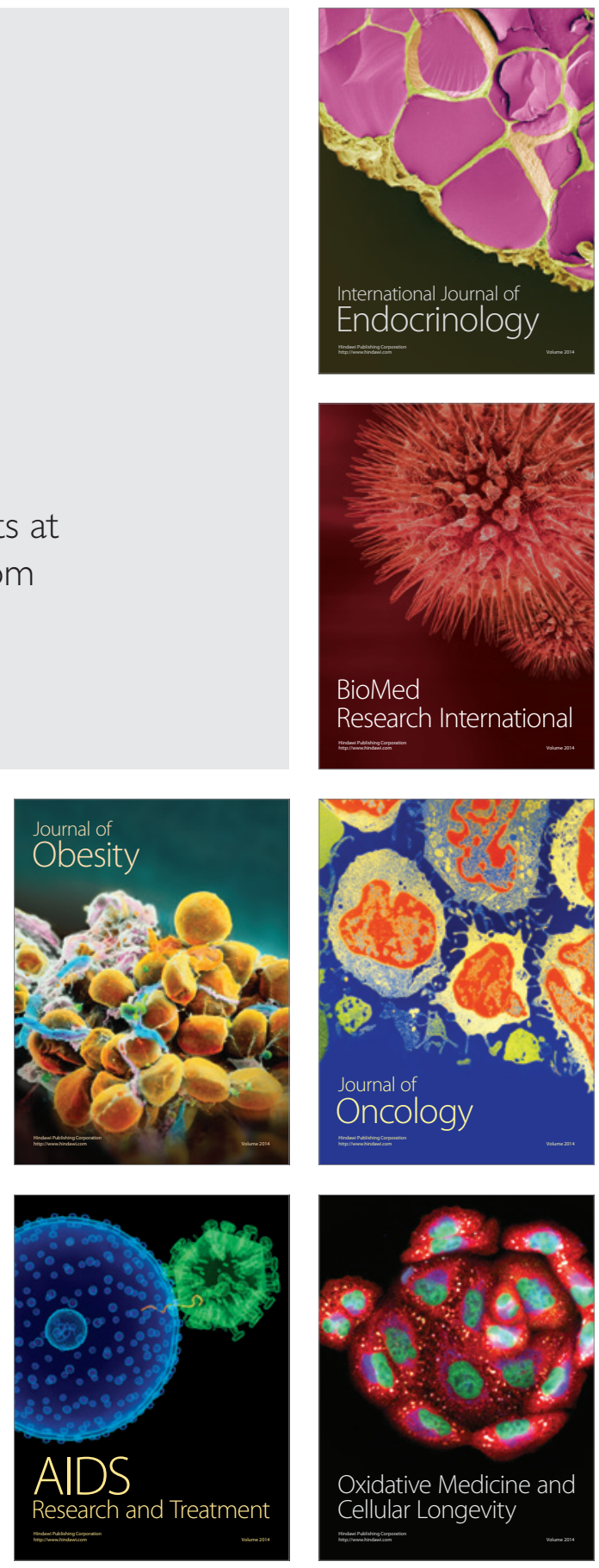